\title{
Propriedades psicométricas da versão em português Brasileiro da Physical Appearance Perfectionism Scale
}

\section{Propiedades psicométricas de la version em português brasileño de La Physical Appearance Perfectionism Scale}

\section{Psychometric Properties of Brazilian Portuguese version of the Physical Appearance Perfectionism Scale}

\author{
Neves, A.N. ${ }^{1}$; Francisco, J.N. ${ }^{2}$; Corazza, J.F. ${ }^{2}$; Carvalho, R.L.P. ${ }^{2}$; Ferreira, L. ${ }^{3}$. \\ ${ }^{1}$ Escola de Educação Física do Exército $;^{2}$ Universidade Sagrado Coração; ${ }^{3}$ Associação Campinense de \\ Psicanálise
}

\begin{abstract}
RESUMO
O perfeccionismo direcionado à aparência física considera haver preocupação com a imperfeição e esperança para a perfeição em relação à aparência. O objetivo dessa pesquisa foi validara versão em português Brasileiro da Physical Appearance Perfectionism Scale em uma amostra de praticantes de exercícios resistidos. A amostra não-probabilística foi constituída por 534 homens e mulheres com idades entre 18 a 39 anos. Foi utilizada análise fatorial confirmatória, para gerar evidências psicométricas para a amostra de referencia. O modelo estrutural bifatorial alcançou índices de ajuste satisfatório, tanto na amostra geral, quanto na amostra por sexo. Em relação à validade convergente, quase todos os valores das cargas fatoriais estavam acima do aceitável, mas a variância média extraída foi adequada apenas na escala masculina. Quanto à validade discriminante, todos os valores da AVE estiveram acima do valor da variância compartilhada nos modelos geral, feminino e masculino, demonstrando haver alguma diferença as duas variáveis latentes. A confiabilidade de constructo foi estabelecida tanto na escala geral, quanto na escala feminina e masculina. O presente instrumento obteve evidências psicométricas adequadas para a versão brasileira da Physical Appearance Perfectionism Scale, o que permite recomendar seu uso no Brasil.
\end{abstract}

Palavras chave: Perfeccionismo; Aparência Física; Imagem Corporal; Psicometria

\section{RESUMEN}

El perfeccionismo dirigido a la apariencia física considera que hay preocupación por La imperfección y la esperanza para la perfección en relación a la apariencia. El objetivo de esta investigación fue validar la versión en portugués brasileño de la Physical Appearance Perfectionism Scale en una muestra de practicantes de ejercicios de resistencia. La muestra no probabilística consistió en 534 hombres y mujeres de entre 18 y 39 años. Se utilizó un análisis factorial confirmatorio, para generar evidencias psicométricas para la muestra de referencia. El modelo estructural bifatorial alcanzó índices de ajuste satisfactorio, tanto en la muestra general, como en la muestra por sexo. En cuanto la validez convergente, case todos los valores de las cargas factorial fueron superiores a los aceptables, aunque la varianza media extraída (AVE) fue adecuada solo en la escala masculina. En cuanto a la validez discriminante, todos los valores AVE estaban por encima del valor de la varianza compartida en los modelos general, femenino y masculino, lo que demuestra que existen algunas diferencias entre las dos variables latentes. La fiabilidad del constructo se estableció tanto en la escala general como en la escala femenina y masculina. Este instrumento obtuvo evidencia psicométrica adecuada para la versión brasileña de Physical Appearance Perfectionism Scale, que permite recomendar su uso en Brasil.

Palabras clave: Perfeccionismo; Apariencia física; Imagen corporal; Psicometría. 


\title{
Nogueira-Neves, A.; Francisco, J.N.; Corazza, J.F.; Carvalho, R.L.P..; Ferreira, L.
}

\begin{abstract}
Perfectionism towards physical appearance considers to be concern about imperfection and hope for perfection in appearance. The aim of this research was to validate the Brazilian Portuguese version of Physical Appearance Perfectionism Scale in a sample of exercise resistance practitioners. The non-probabilistic sample consisted of 534 men and women aged 18 to 39 years. Confirmatory factorial analysis was used to generate psychometric evidence for the reference sample. The two-factor structural model achieved satisfactory adjustment indices, both in the general sample and in the sample by sex. Regarding convergent validity, almost all values of factorial loads were above acceptable, although the average variance extracted (AVE) was adequate only in the male scale. Regarding the discriminant validity, all the AVE values were above the value of the shared variance in the general, female and male models, showing that there are some differences between the two latent variables. Construct reliability was established on both the general scale and the female and male scale. This instrument achieved adequate psychometric evidence for the Brazilian version of Physical Appearance Perfectionism Scale, which allows recommending its use in Brazil.
\end{abstract}

Keywords: Perfectionism; Physical appearance; Body image; Psychometry.

\section{INTRODUÇÃO}

A aparência física é uma característica pessoal óbvia e acessível aos outros no processo de interação social. Tendo essa função, o binômio beleza-bom - e seu consequente antônimo feiura-mau - é um valor associado à aparência, identificado na década de 1970 (Dion, Bercheid, \&Walster, 1972). Mesmo havendo evidências de que há outros esteriótipos associados à aparência do corpo, como beleza - egoísmo e beleza atração sexual (Cash \& Duncan, 1984; Dermer\&Thiel, 1975; Deaux\& Lewis, 1984; Gillen, 1981), parece que beleza-bom ainda é majoritário em nossa sociedade ocidental (Hennessy, Fishbein, Curtis, \& Barrett, 2008). Para as mulheres, belo é o corpo magro, a pele clara, cabelos lisos e olhos, de preferência, claros também (Grogan, 2008; Vigarello, 2006). Para os homens, a beleza concretiza-se num corpo alto, musculoso e forte, com baixo percentual de gordura, ombros largos e rosto anguloso (Adams, Truner, \&Bucks, 2005; Schwartz \&Tylka, 2008)

Cirurgia plástica, tratamentos cosméticos diversos - para pele, cabelos, dentes - dietas e exercícios físicos são intervenções que podem ser realizadas para transformação do corpo e manipulação da aparência para tentar atender ao padrão de beleza social vigente. Expectativas irreais a respeito do corpo, em adição ao traço de personalidade perfeccionista estão associadas com insatisfação corporal, ansiedade físico social e dismorfia muscular, sugerindo que o perfeccionismo se evidencia no corpo (Haase, Prapavessis, \& Owens, 2002; Hanstock \& O‘Mahony, 2002; Ruggeiro, Levi, Ciuna, \& Sassaroli, 2003). O perfeccionismo ainda está relacionado aos transtornos alimentares, aos exercícios resistidos, ao excesso de exercício e às cirurgias plásticas cosméticas (Davis \& Scott-Robertson, 2000; Gulker, Laskis, \& Kuba, 2001; Cockell et al., 2002; Hewitt, Sherry, Flett, \&Shick, 2003). O exercício físico é um recurso comum entre homens para alteração da aparência, enquanto a dieta alimentar parece ser a escolha primeira para as mulheres (Cash \& Smolak, 2011). Apesar do exercício físico ser reconhecido como um comportamento saudável (Hardman \& Stensel, 2009), já foi demonstrado que excesso de exercício físico e altas cargas, ocasionados por um comportamento perfeccionista e uma busca obsessiva para melhorar a aparência física pode impactar a saúde do indivíduo (Haase, Prapavessis, \& Owens, 2002; Hanstock \& O'Mahony, 2002).

É nessa necessidade de atender à um padrão de corpo ideal e a busca incessante por isso, que se considera o perfeccionismo relacionado à aparência. Pode ser definido como uma forma de domínioespecífico do perfeccionismo que compreende dois componentes: esperança de perfeição e preocupação com a imperfeição (Yang \& Stoeber, 2012). Esperança de perfeição se relaciona com os esforços perfeccionistas para conquistar a aparência física desejada, ideal. Compreende o esforço de autoorientação, estabelecimento de altos padrões pessoais e a necessidade de parecer perfeito (Yang \& Stoeber, 2012). Já a preocupação com a imperfeição versa sobre os aspectos de preocupação com as falhas ainda existentes e o julgamento externo e capta o perfeccionismo socialmente prescrito, crítico, autoavaliativo, as preocupações acerca da avaliação 


\section{Validação da PAPS no Brasil}

dos outros, a necessidade de evitar a imperfeição (Bieling et al., 2004; Stoeber \& Yang, 2015).

No contexto do exercício físico e esporte, há hoje disponível no Brasil a Sport Multidimensional Perfectionism Scale-2 (Nascimento Jr, Vissoci, Lavallee \& Vieira 2015), que avalia as tendências perfeccionistas de atletas. Faltava ainda um instrumento com propriedades psicométricas adequadas para avaliar o perfeccionismo da aparência no praticante de exercícios comum (não-atleta) que pudesse ser usado nas avaliações do impacto da prática de exercícios físicos na saúde. Considerando as evidências acerca do perfeccionismo da aparência e exercício físico, Ferreira, Corazza, Francisco e Neves (in press) fizeram um pequeno avanço nesse sentido, ao adaptar transculturalmente a Physical Appearance Perfectionism Scale (PAPS). A PAPS considera em seu modelo estrutural os dois fatores - esperança de perfeição e preocupação com a imperfeição teoricamente previstos, que foram confirmados em seu estudo de desenvolvimento (Yang \& Stoeber, 2012).

Apenas adaptação cultural não garante que o instrumento seja válido e confiável. Por isso, para que o PAPS possa ser usada no Brasil, é necessária uma continuidade da pesquisa metodológica de Ferreira et al. (in press), destinada à verificação das qualidades psicométricas do instrumento. Mais especificamente, com as propriedades psicométricas do instrumento dizem respeito à amostra de referência (Marôco, 2014), para que seja possível usar a PAPS em investigações acerca do perfeccionismo da aparência e exercício físico, a amostra deve ser praticante de algum tipo de exercício. O estudo de validação psicométrica, com investigação de evidências de validade de constructo e confiabilidade interna em uma amostra de brasileiros de ambos os sexos praticantes de exercício resistidos é o que vem a ser o objetivo do presente estudo.

\section{MATERIAL E MÉTODOS}

Este é um estudo metodológico com amostragem não probabilística. Foi autorizado pelo Comitê de Ética e Pesquisa da Universidade do Sagrado Coração sob o parecer $n^{\circ}$ 1.734.372.

\section{Desenho de estudo e tamanho amostral}

O tamanho amostral foi calculado de acordo com as recomendações de Hair, Anderson, Tatham, \& Black (2009). Os autores preconizam que o tamanho amostral deve ser de $5-10(\mathrm{k})$ participantes por parâmetro avaliado. Considerando que a PAPS tem 26 parâmetros (12 variáveis observáveis; 12 erros; 2 variáveis latentes de primeira ordem) e $\mathrm{k}=10$ (valor máximo para essa constante), o número amostral mínimo para esta pesquisa era de 260 participantes.

A amostra foi composta por 534 participantes (mulheres $=50.6 \%, \mathrm{n}=270$ ). A média da idade das mulheres foi de $24.88 \pm 5.33$, variando entre 18 e 39 anos. Do total, 36.7\% $(\mathrm{n}=99)$ se declararam solteiras, sem namorado; $42.6 \%(\mathrm{n}=115)$ namorando, $18.9 \%$ $(\mathrm{n}=51)$ casadas e apenas $1.9 \%(\mathrm{n}=5)$ eram divorciadas. Em relação à prática de exercício resistido, a maioria, $70.4 \%(\mathrm{n}=190)$ praticava musculação entre 1 e 3 anos, a demais $(29.6 \%, \mathrm{n}=80)$ entre 3 e 9 anos ou mais. A maioria praticava 3 sessões por semana $(45.9 \%$, $\mathrm{n}=124$ ), com a duração da sessão entre 1 e 2 horas (94.4\%, $\mathrm{n}=255)$, com intensidade variando entre leve $(26.3 \%, \mathrm{n}=44)$, leve a moderado $(26.3 \%, \mathrm{n}=71)$, moderado (34.8\%, $\mathrm{n}=94)$, moderado e pesado $(20.4 \%$, $\mathrm{n}=55)$ e pesado $(2.2 \%, \mathrm{n}=6) . \quad$ Em relação à alimentação, $14.8 \%(\mathrm{n}=40)$ disseram estar em dieta para ganhar peso, $40 \%(\mathrm{n}=108)$ em dieta para perder peso e $45.2 \%$ (n=122) disseram não estar de dieta.

Entre os homens, as idades máxima e mínima foram iguais às das mulheres e a média foi de $25.55 \pm$ 5.14 anos. Quanto ao estado civil, $43.2 \%(\mathrm{n}=114)$ dos homens se declarou solteiro (sem namorada), 37.5\% $(n=99)$ disseram estar namorando, 16.7\% $(n=44)$ casados e os demais divorciados $(2.7 \%, \mathrm{n}=7)$. Em relação à prática de musculação, $42.8 \%(\mathrm{n}=113) \mathrm{o}$ faziam de 1 a 3 anos; $24.6 \%(n=65)$ de 3 a 5 anos e os demais $(32.6 \%, \mathrm{n}=86)$ o faziam de 5 a mais de 9 anos. A maioria, $41.3 \%(n=109)$, faz musculação 5 vezes na semana e $93.2 \%(n=246)$ tem a sessão durando de 1 a 2 horas, sendo a intensidade moderada a pesada a mais frequente, $39.4 \%(n=104)$. Em relação a adoção de dieta, $32.2 \%(\mathrm{n}=85)$ disseram estar em dieta para ganhar peso, $23.5 \%(\mathrm{n}=62)$ em dieta para perder peso e $44.3 \%(n=117)$ não estava fazendo qualquer dieta.

\section{Instrumentos}

Versão Brasileira da Physical Appearance Perfectionism Scale (PAPS; Yang \& Stoeber, 2012; adaptação transcultural: Ferreira et al., in press). A PAPS Foi desenvolvida para avaliar aspectos positivos e negativos do perfeccionismo relacionado à aparência física (Yang \& Stoeber, 2012). A escala original tem 12 itens, organizados numa escala Likert de 5 pontos 


\section{Nogueira-Neves, A.; Francisco, J.N.; Corazza, J.F.; Carvalho, R.L.P..; Ferreira, L.}

$(1=$ discordo fortemente; 5 = concordo fortemente $)$. Maiores pontuações indicam maior grau de perfeccionismo. A escala original está operacionalizada em 2 fatores: (1) Preocupações com a Imperfeição (PI), itens $1,3,5,8,9,10$ e 11 e (2) Esperança para perfeição (EP), itens 2, 4, 6, 7 e 12 (Yang \& Stoeber, 2012).

Versão Brasileira da Social Physique Anxiety Scale (SPAS; Hart, Leary, \& Rejesky, 1989; versão Brasileira: Neves, Neves, Zanetti, Brandão, \& Ferreira, 2018). A SPAS é uma escala originalmente unifatorial de 12 itens, criada para avaliar a ansiedade que se pode experimentar em resposta à avaliação de outra pessoa sobre seu físico/aparência. As respostas das escalas estão dispostas numa escala tipo Likert de cinco pontos, que variam de 1 = nada parecido comigo a 5 = tudo a ver comigo. O escore final da escala é dado pela soma de todos os itens. Quanto maior a soma do escore, maior é o grau de ansiedade frente ao olhar do outro. No Brasil, Neves et al. (2018), verificaram estrutura fatorial distinta para a SPAS para homens e para mulheres. Para a amostra feminina, o modelo com melhor ajuste foi unidimensional com 10 itens $(1,2,3$, $4,5,6,7,9,10,12) ;$ RMSEA $=.065$, GFI $=.99$, AGFI $=.99, \mathrm{NFI}=.99, \mathrm{CFI}=1, \mathrm{NNFI}=1$; e para a amostra masculina o melhor ajuste ocorreu no modelo unidimensional de 9 itens $(1,2,3,4,5,7,9,10,12)$; RMSEA $=.076$, GFI $=.99$, AGFI $=.98$, NFI $=.98$, CFI $=.99$, NNFI $=.99$. Na amostra deste estudo, o alpha de Cronbach foi de $\alpha=.89$ para a amostra feminina e $\alpha=.85$ para a amostra masculina. Este instrumento foi utilizado para análise da validade nomológica PAPS, testando a hipótese de associação entre ansiedade físico social e perfeccionismo da aparência.

\section{Versão Brasileira da Exercise Dependence Scale-} Revised (EDS-R; Downs, Hausenblas, \& Niggs, 2004; versão Brasileira: Alchieri, Gouveia, Oliveira, \& Diógenes, 2015). Elaborada para avaliar o grau de dependência do exercício, com base nos critérios de dependência de transtornos, relacionados ou não a substâncias, do Diagnostic Statistics Manual of Mental Disorders - $I V$. As opções de respostas estão dispostas numa escala tipo Likert, de 6 pontos, que variam de $1=$ nunca a $6=$ sempre. Para obter o resultado da escala, some todos os itens: quanto maior a pontuação, maior é a tendência a ser dependente do exercício. A versão Brasileira da escala é composta pelos 21 itens originais, operacionalizados em 7 fatores, obtidos através de análise fatorial exploratória: (1) Intencionalidade (I-EDS) itens 7, 14 e 21; (2) Continuidade (C-EDS) itens 2, 9 e 16; (3) Tolerância (To-EDS) itens 3, 10 e 17; (4) Redução de outras atividades (R-EDS) itens 5, 12 e 19; (5) Falta de Controle (FC-EDS) itens 4, 11 e 18; (6) Abstinência (A-EDS) itens 1,8 e 15 e (7) Tempo (Te-EDS) itens 6,13 e 20. Os 7 fatores explicaram $62 \%$ da variância da versão Brasileira do instrumento. Nessa pesquisa, os valores de alpha de Cronbach dos fatores variaram entre $\alpha=.75-.91$ para mulheres e $\alpha=.72-.92$ para amostra masculina. Este instrumento foi utilizado para análise da validade nomológica PAPS, testando a hipótese de associação entre a dependência de exercício e perfeccionismo da aparência.

Questionário demográfico: especialmente elaborado para esta pesquisa, no questionário demográfico foram inseridas perguntas a respeito da idade, estado civil, escolaridade, pratica de exercícios resistidos -tempo, intensidade, duração e frequência.

\section{Procedimentos e Aspectos Éticos}

Inicialmente, foram visitadas academias no interior do estado de São Paulo. Seus diretores/proprietários foram consultados quanto à possibilidade de realizar coleta de dados em seu estabelecimento. Frente à permissão, os alunos da academia eram abordados ao final de seu treino, na saída da academia, momento em que era explicado oralmente os objetivos da pesquisa. Somente para aqueles que concordaram em participar voluntariamente foi entregue o pacote de questionários como Termo de Consentimento Livre e Esclarecido. O preenchimento dos questionários foi feito na própria academia, em mesas e em espaço privado providenciado para a coleta de dados. Os participantes preencheram os instrumentos de forma individual, levando entre 15 a 25 minutos para terminar.

\section{Modelos investigados}

Além do estudo de desenvolvimento e investigação psicométrica inicial (Yang \& Stoeber, 2012), não houve qualquer outro estudo de validação psicométrica da PAPS. Na inspeção do conteúdo das variáveis observáveis, não se destaca nenhuma outra variável latente que possa ser representada pelos itens, 


\section{Validação da PAPS no Brasil}

além das duas previstas no estudo original. Assim, no presente estudo, investigou-se a adequação dos dados amostrais à estrutura fatorial original da PAPS, descrita anteriormente (Yang \& Stoeber, 2012) (Figura 1).

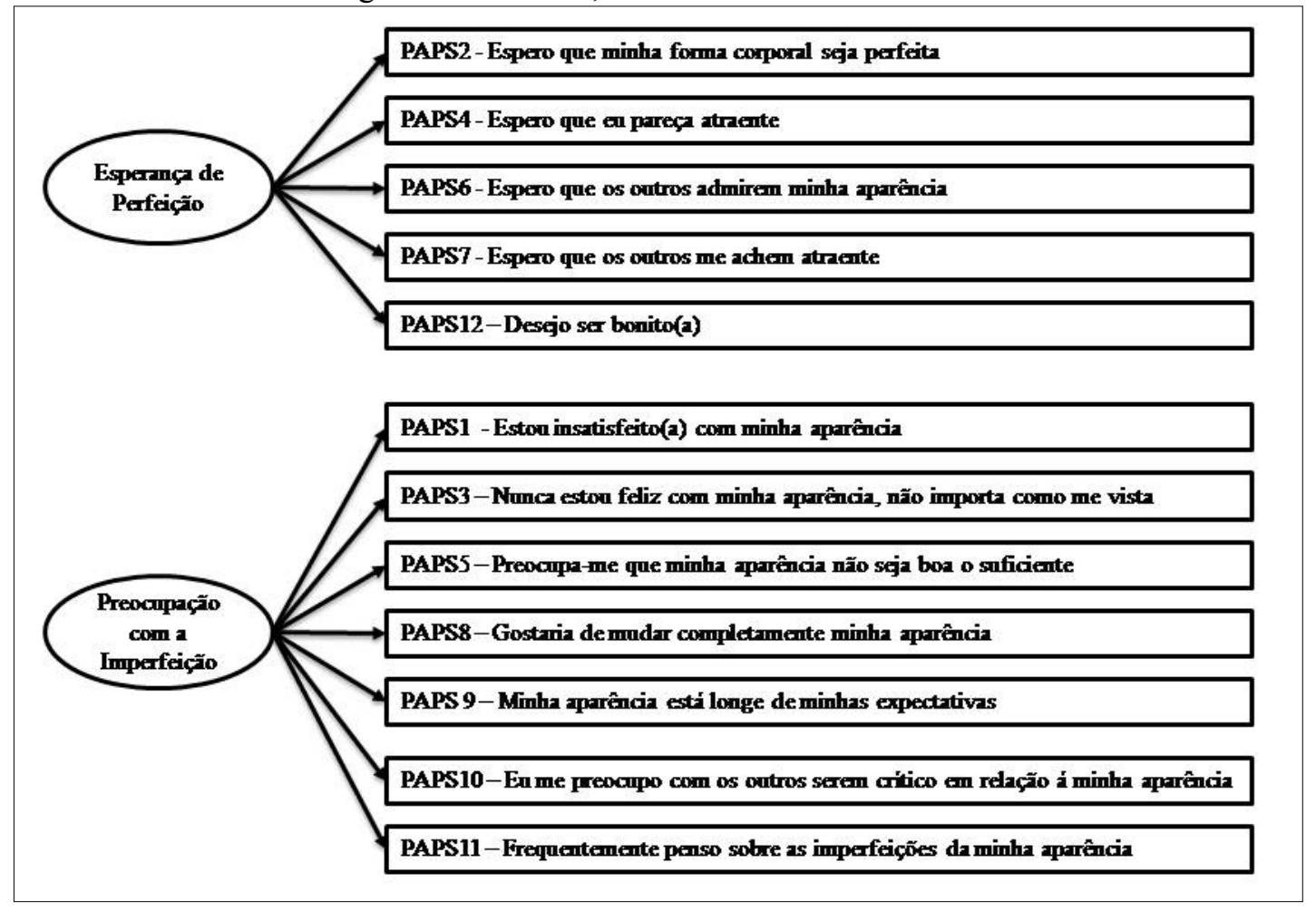

Figura 1 - Modelo estrutural original da PAPS

\section{Análise estatística}

Para preparar os dados para a Análise Fatorial Confirmatória (AFC), foi usado o módulo PRELIS ${ }^{\mathrm{TM}_{2}}$ do sistema LISREL ${ }^{\circledR}$. Subsequentemente, foi usado o modelo SIMPLIS, para a geração da AFC. Como a distribuição dos dados não foi aderente à distribuição normal multivariada, o método de extração Unweighted Least Square foi usado por não ser sensível à essa violação (Garson, 2006). Para os dados faltantes, foi adotado o critério de deleção listwise (Jöreskog \& Sörbom, 1999).

Para avaliação do ajuste do modelo estrutural, foram considerados os seguintes índices de ajuste: Goodness-of-Fit Index (GFI), Adjusted Goodness-ofFit Index (AGFI), Normed Fit Index (NFI), NonNormed Fit index (NNFI) e Comparative Fit Index (CFI). Foi também considerado o Root Mean Square Error of Approximation (RMSEA). O modelo foi considerado satisfatório quando $\chi^{2} / g l \leq 5$; RMSEA $\leq .08$; CFI, NFI, NNFI, GFI e AGFI $\geq .95$ (Hair et al.,
2009). O refinamento do modelo foi feito a partir da análise das cargas fatoriais, resíduos e os índices de modificação do sistema LISREL ${ }^{\circledR}$. Cada modificação no modelo foi feita individualmente e apenas uma a cada nova rotação do modelo.

$\mathrm{Na}$ análise do modelo de mensuração, considerou-se a validade de constructo (convergente, discriminante e nomológica) e a consistência interna.

Para estabelecer a validade convergente, foram considerados como evidências as cargas fatoriais e a Variância Média Extraída (AVE). Cargas fatoriais $(\lambda)$ acima de .75 são preferíveis e acima de .50 são aceitáveis (Hair et al., 2009). A AVE foi calculada pela fórmula que se segue:

$$
A V E=\frac{\left(\sum \lambda_{j}{ }^{2}\right)}{\left(\Sigma \lambda_{j}{ }^{2}\right)+\sum E_{j}}
$$

em que $\lambda \mathrm{j}$ é a carga fatorial padronizada do indicador (assertiva) $j$ e $E_{j}$ é o erro de mensuração do 


\section{Nogueira-Neves, A.; Francisco, J.N.; Corazza, J.F.; Carvalho, R.L.P..; Ferreira, L.}

indicador $\mathrm{j}$, calculado como 1- confiabilidade do indicador. Valores acima de .50 são considerados aceitáveis (Hair et al., 2009).

A validade discriminante foi avaliada através da comparação da variância compartilhada $\left(\mathrm{r}^{2}\right) \operatorname{com}$ a AVE de cada variável latente. A evidência da validade discriminante é estabelecida quando a $\mathrm{AVE} \geq .50 \mathrm{e}$ maior que a variância compartilhada entre os fatores (Fornell \& Lacker, 1981).

A validade nomológica foi estimada através da associação entre os escores dos fatores da PAPS, EDS e SPAS, testando as hipóteses de que no Brasil a associação entre perfeccionismo da aparência, ansiedade físico social e dependência do exercício se confirma, a exemplo de estudos anteriores (Hagan \& Hausenblas, 2003; Sabiston , Pila, PinsonnaultBilodeau, \& Cox, 2014; Mohammed, 2017).

Para estabelecer a confiabilidade interna, o alpha de Cronbach $(\alpha)$ e a confiabilidade composta (CC) foram calculados, sendo considerados aceitáveis valores acima de .70 para os dois parâmetros. (Nunnaly, 1978; Hair et al., 2009). A confiabilidade composta foi calculada pela fórmula:

$$
C C=\frac{\left(\Sigma \lambda_{\mathrm{j}}\right)^{2}}{\left(\Sigma \lambda_{\mathrm{j}}\right)^{2}+\Sigma E_{j}}
$$

em que $\lambda \mathrm{j}$ é a carga fatorial padronizada do indicador (assertiva) $j$ e $E$ é o erro de mensuração do indicador $\mathrm{j}$, calculado como 1- confiabilidade do indicador.

O método da Máxima Verossimilhança (ML) foi empregado para verificar a não-invariância dos modelos para cada sexo. O teste de invariância foi realizado por análise multigrupo usando a diferença de qui-quadrado $\left(\Delta \chi^{2}\right)$ entre os níveis de invariância. $\mathrm{O}$ teste foi realizado considerando a análise das cargas fatoriais ( $\lambda$, interceptos (I) e resíduos de variância/covariância (Marôco, 2014). Seguindo as recomendações de Jöreskog e Soborn (1999) e Brown (2014) seguimos os seguintes passos: (1) ajustamos o modelo separadamente para a amostra feminina $(\mathrm{n}=270)$ e masculina $(\mathrm{n}=264)$; (2) verificamos a invariância configural (forma); (3) verificamos a invariância métrica e (4) verificamos a variância escalar e (5) verificamos a invariância estrita. A invariância métrica foi suportada se $p \Delta \chi_{\lambda}^{2}>.05$. A invariancia métrica e escalar foi suportada se $p \Delta \chi_{\lambda}^{2}{ }_{\lambda} \mathrm{e} p \Delta \chi_{\mathrm{I}_{\mathrm{I}}}^{2}>$.05. Finalmente, a invariância estrita foi confirmada se $p \Delta \chi_{\lambda}^{2}, p \Delta \chi_{\mathrm{I}}^{2}$, and $p \Delta \chi_{\text {cov/res }}^{2}>05$ (Kaplan, 2000; Marôco, 2014).

\section{RESULTADOS}

\section{Estrutura fatorial}

A primeira estimação do modelo mostrou índices pobres de ajuste, $\chi^{2} / g l=6.96, \mathrm{RMSEA}=.106$, $\mathrm{CFI}=.95, \mathrm{NFI}=.93, \mathrm{NNFI}=.93, \mathrm{GFI}=.97, \mathrm{AGFI}$ $=.95$. Para melhor ajuste do modelo, foram retirados os itens 1 e 2 por suas baixas cargas fatoriais, $\lambda=.21$ e $\lambda=.35$ respectivamente. $\mathrm{Na}$ inspeção dos índices de modificação do LISREL a covariância dos erros entre os itens 7 e 6,9 e 8,9 e 3 tinham coerência conceitual e por isso foram inseridos no modelo. Cada modificação ocorreu a cada nova estimação. O modelo final alcançou ajuste satisfatório, $\chi^{2} / g l=4.22$, $\mathrm{RMSEA}=.078, \mathrm{CFI}=.97, \mathrm{NFI}=.96, \mathrm{NNFI}=.96, \mathrm{GFI}$ $=.98, \mathrm{AGFI}=.97($ Figura 2A) .

Para a amostra feminina $\left(\chi^{2} / g l=4.45\right.$, $\mathrm{RMSEA}=.113, \mathrm{CFI}=.94, \mathrm{NFI}=.92, \mathrm{NNFI}=.93, \mathrm{GFI}$ $=.96, \mathrm{AGFI}=.94) \mathrm{e}$ masculina $\left(\chi^{2} / g l=3.64\right.$, $\mathrm{RMSEA}=.100, \mathrm{CFI}=.96, \mathrm{NFI}=.93, \mathrm{NNFI}=.95, \mathrm{GFI}$ $=.96, \mathrm{AGFI}=.95)$, a estimação inicial foi igualmente inadequada para alguns parâmetros de ajuste. Adotando as mesmas alterações realizadas para o ajuste da amostra total, foi obtido ajuste satisfatório tanto para mulheres quanto para homens, $\chi^{2} / g l=2.53$, $\mathrm{RMSEA}=.075, \mathrm{CFI}=.98, \mathrm{NFI}=.96, \mathrm{NNFI}=.97, \mathrm{GFI}$ $=.98, \mathrm{AGFI}=0,96 \mathrm{e} \chi^{2} / g l=2.72, \mathrm{RMSEA}=.081, \mathrm{CFI}$ $=.98, \mathrm{NFI}=.96, \mathrm{NNFI}=.97, \mathrm{GFI}=.98, \mathrm{AGFI}=.96$, respectivamente (Figuras2A, $2 \mathrm{~B}$ e $2 \mathrm{C}$; tabela 1 ). 


\section{Validação da PAPS no Brasil}

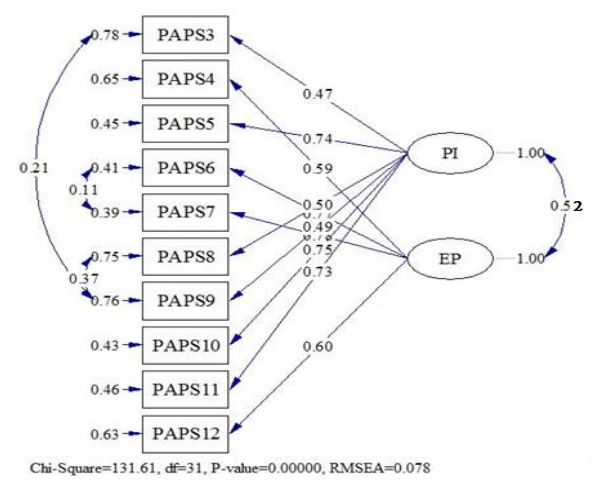

$\mathbf{A}$

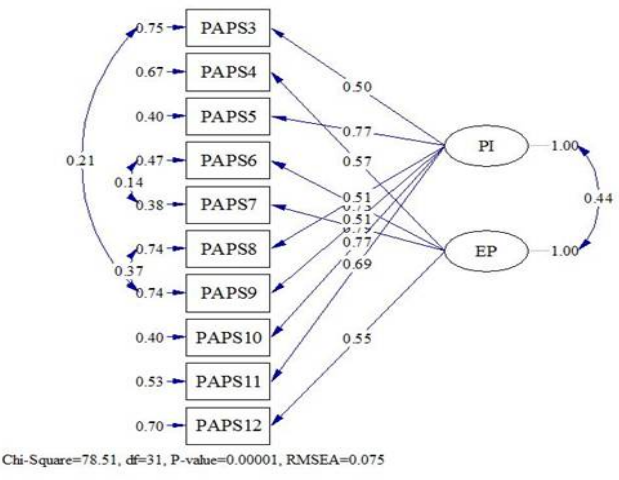

B

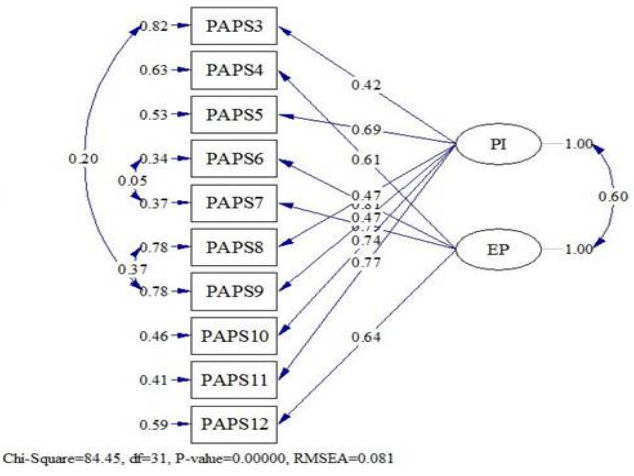

C

Figura 2: Modelos ajustados da PAPS para amostras geral (A), amostra feminina (B) e amostra masculina (C)

Tabela 1. Índices de ajuste do modelo de medida e mensuração para a PAPS nas três amostras

\begin{tabular}{|c|c|c|c|c|c|c|c|c|c|c|c|c|}
\hline Amostra & Modelo & $\mathrm{n}$ & $\Lambda$ & $\chi^{2 / g 1}$ & CFI & NFI & GF & RMSEA & $\alpha$ & $\mathrm{CC}$ & AVE & $\mathrm{R}^{2}$ \\
\hline \multirow{2}{*}{ Total } & Inicial & \multirow{2}{*}{534} & $\overline{.49}$ & $\overline{6.96}$ & $\overline{.95}$ & .93 & $\overline{.97}$ & .106 & $\overline{.77 \text { e } .79}$ & .78 e .80 & .37 e .43 & .24 \\
\hline & Ajustado & & .52 & 4.22 & .97 & .96 & .98 & .078 & .79 e .81 & .78 e .79 & .39 e .48 & .27 \\
\hline \multirow{2}{*}{ Feminina } & Inicial & \multirow{2}{*}{270} & .42 & 4.45 & .94 & .92 & .96 & .113 & $.75 \mathrm{e} .80$ & .77 e .80 & $.41 \mathrm{e} .41$ & .18 \\
\hline & Ajustado & & .44 & 2.53 & .98 & .96 & .98 & .075 & .76 e. 82 & .76 e .81 & $.41 \mathrm{e} .45$ & .19 \\
\hline \multirow{2}{*}{ Masculina } & Inicial & \multirow{2}{*}{264} & .56 & 3.64 & .96 & .93 & .96 & .100 & .78 e .79 & .78 e .80 & .35 e .45 & .31 \\
\hline & Ajustado & & .60 & 2.72 & .98 & .96 & .98 & .081 & .80 e .81 & $.77 \mathrm{e} .81$ & .37 e .52 & .36 \\
\hline
\end{tabular}

Nota: $\lambda=$ cargas fatoriais, $\chi^{2} / g l=$ Qui-Quadrado Normado, CFI $=$ Comparative Fit Index, NFI $=$ Normed Fit Index, RMSEA $=$

Root Mean Square Error of Approximation, $\alpha=$ Cronbach's alpha; CC = Confiabilidade Composta; AVE =Variância Média Extraída; $R^{2}=$ variância compartilhada. *

\section{Validade convergente e discriminante}

Em relação à validade convergente, considerando a AVE, a mesma pode ser evidenciada apenas para o fator EP na amostra masculina (AVE $=.52$ ). Nos demais fatores e amostras, o valor de AVE foi abaixo do recomendado (tabela 2). Ressaltamos apenas que os itens 3 e 9 obtiveram carga fatorial abaixo do valor recomendado na escala geral $(\lambda=.47$ e $\lambda=.49)$ e masculina $(\lambda=.42$ e $\lambda=.47)$; os itens 8 $(\lambda=.47)$ apenas na escala masculina. Todavia, como estavam próximos desse valor foram mantidos no modelo, com o propósito de assegurar o conteúdo original do instrumento.

Em relação à validade discriminante, todos os valores de AVE estiveram acima do valor da variância compartilhada $\left(\mathrm{r}^{2}\right)$ nos três modelos, demonstrando haver alguma diferença entre as duas variáveis latentes (tabela 2).

\section{Validade nomológica}

$\mathrm{Na}$ amostra feminina e masculina, confirmamos parcialmente a hipótese de haver associação entre a PAPS e a EDS. Nas duas amostras, todos os fatores, com exceção do fator Abstinência da EDS tiveram associações com o fator Preocupação com a Imperfeição. $\mathrm{Na}$ amostra feminina, apenas os fatores Tolerância e Falta de Controle tiveram correlações significantes com o fator Esperança para perfeição. $\mathrm{Na}$ amostra masculina, apenas os fatores Abstinência e Continuidade não foram correlacionados com o fator

Esperança para perfeição. Em relação à SPAS, a hipótese foi totalmente confirmada, já que ambos os fatores da PAPS, tanto na amostra masculina como feminina foram significantemente correlacionados com o escore da SPAS (tabela 3 ). 


\section{Nogueira-Neves, A.; Francisco, J.N.; Corazza, J.F.; Carvalho, R.L.P..; Ferreira, L.}

Tabela 2 - Valores da AVE, correlação entre fatores e variância compartilhada dos fatores da PAPS para cada amostra

\begin{tabular}{llllllc}
\hline Fator & \multicolumn{2}{l}{ Amostra total } & \multicolumn{2}{c}{ Amostra feminina } & \multicolumn{2}{c}{ Amostra masculina } \\
& PI & EP & PI & EP & PI & EP \\
\hline PI & $\mathbf{. 3 9}$ & .52 &. $\mathbf{4 1}$ & .44 & .37 & .49 \\
EP & .27 &. $\mathbf{4 8}$ & .19 & $\mathbf{. 4 5}$ & .24 & $\mathbf{. 5 2}$
\end{tabular}

Nota: $P I=$ fator preocupação com a Imperfeição; $E P$ = Esperança para perfeição. Na linha diagonal em negrito estão dispostos os valores da AVE. Acima da linha diagonal, os valores de correlação entre os fatores. Abaixo da linha diagonal, os valores da variância compartilhada

Tabela 3 - Correlações entre fatores da PAPS, EDS e perguntas diretas

\begin{tabular}{|c|c|c|c|c|c|c|c|c|c|c|c|c|}
\hline & PI & EP & I & $\mathrm{C}$ & To & ROA & $\mathrm{FC}$ & $\mathrm{A}$ & $\mathrm{Te}$ & SPAS & $\mathrm{P} 1$ & $\mathrm{P} 2$ \\
\hline PI & & $.31 * *$ & $.18 * *$ & $.17 * *$ & $.16 * *$ & $.21 * *$ & $.12 *$ & .08 & $.15^{*}$ & $.57 * *$ & $.46 * *$ & -.02 \\
\hline EP & $.44 * *$ & & .05 & .05 & $.12 *$ & .02 & $.13^{*}$ & .09 & -.01 & $.34 * *$ & .16 & .01 \\
\hline I & $.25^{* *}$ & $.13^{*}$ & & $.43 * *$ & $.47 * *$ & $.56^{* *}$ & $.64 * *$ & .32 & $.77 * *$ & .12 & $.26^{* *}$ & $.37 * *$ \\
\hline $\mathrm{C}$ & $.21 * *$ & .08 & $.57 * *$ & & $.31 * *$ & $.46^{* *}$ & $.45^{* *}$ & $.26 * *$ & $.48 * *$ & $.21 * *$ & $.26 * *$ & $.25 * *$ \\
\hline To & $.15^{*}$ & $.21 * *$ & $.48 * *$ & $.49 * *$ & & $.41 * *$ & $.69 * *$ & $.45 * *$ & $.52 * *$ & .10 & $.33 * *$ & $.43 * *$ \\
\hline ROA & $.34 * *$ & $.21 * *$ & $.48 * *$ & $.51 * *$ & $.51 * *$ & & $.49 * *$ & $.70 * *$ & $.61 * *$ & $.15^{*}$ & $.27 * *$ & $.29 * *$ \\
\hline $\mathrm{FC}$ & $.15^{*}$ & $.20 * *$ & $.38 * *$ & $.30 * *$ & $.64 * *$ & .44 & & $.49 * *$ & $.59 * * *$ & $.14 *$ & $.38 * *$ & $.50 * *$ \\
\hline A & .06 & .11 & $.41 *$ & $.38 * *$ & $.40 * *$ & $.38 * *$ & $.42 * *$ & & $.38 * *$ & .11 & $.31 * *$ & $.29 * *$ \\
\hline $\mathrm{Te}$ & $.25 * *$ & $.19 * *$ & $.79 * *$ & $.54 * *$ & $.57 * *$ & $.64 * *$ & $.49 * *$ & $.45^{* *}$ & & .09 & $.29 * *$ & $.36^{* *}$ \\
\hline SPAS & $.45^{* *}$ & $.34 * *$ & $.18 * *$ & $.21 * *$ & $.20 * *$ & $.32 * *$ & $.15^{*}$ & $.14^{*}$ & $.25 * *$ & & $.45 * *$ & .01 \\
\hline $\mathrm{P} 1$ & .10 & $.14^{*}$ & $.31 * *$ & $.27 * *$ & $.41 * *$ & $.30 * *$ & $.56 * *$ & $.23 * *$ & $.42 * *$ & .09 & & $.32 * *$ \\
\hline $\mathrm{P} 2$ & $.41 * *$ & $.31 * *$ & $.31 * *$ & $.35 * *$ & $.29 * *$ & $.44 * *$ & $.30 * *$ & $.19 * *$ & $.40 * *$ & $.47 * *$ & $.28 * *$ & \\
\hline
\end{tabular}

Nota: $P I=$ fator preocupação coma imperfeição da PAPS, EP = fator esperança para perfeição da $P A P S, I=$ fator intencionalidade da $E D S, C=$ fator continuidade da EDS, To = fator tolerância da EDS, ROA = fator redução de outras atividades da EDS, FC = fator falta de controle da EDS, Te = fator tempo da EDS. SPAS: ansiedade físico social, P1 = pergunta direta 1: "Indique numa escala de 0 a 10 qual o seu nível de esforço para alcançar resultados satisfatórios elou altos desempenhos com a aparência física", P2 = pergunta direta 2: "Indique numa escala de 0 a 10 o quanto não atingir o nível de desempenho desejado ou ideal em relação a aparência te deixa ansioso." ** correlações significantes a .001. * Correlações significantes a .05. Valores acima da linha diagonal referem-se à amostra feminina e abaixo da linha diagonal à amostra masculina.

\section{Confiabilidade interna}

Os valores de confiabilidade composta ficaram acima de .50 , tanto na escala geral $(\mathrm{PI}=.79 \mathrm{e} \mathrm{EP}=.78)$ quanto na escala feminina $(\mathrm{PI}=.81$ e $\mathrm{EP}=.76) \mathrm{e}$ masculina $(\mathrm{PI}=.77$ e $\mathrm{EP}=.81)$. Igualmente, foram adequados os valores de alpha de Cronbach para o fator PI tanto para a amostra geral $(\alpha=.81)$ quanto para a amostra feminina $(\alpha=.82)$ e masculina $(\alpha$ $=.80$ ), assim como os valores do fator EP, $\operatorname{com} \alpha=.79$ para a amostra geral, $\alpha=.76$ para a amostra feminina e $\alpha=.81$ para a amostra masculina.
Não-invariância por sexo

$\mathrm{O}$ teste de não-invariância entre homens e mulheres não foi suportado na não-invariância métrica, $\Delta \chi_{\lambda}^{2}(11)=41.6, p=.001$; consequentemente, nem na escala $\Delta \chi_{I}^{2}(18)=34,66, p=.01$; $\mathrm{e}$ estrita $\Delta \chi_{\text {cov/res }}^{2}(13)=20.67, p=.08$ (tabela 4$)$.

\section{DISCUSSÃO}

O objetivo deste estudo foi validar psicometricamente a versão em Língua Portuguesa do Brasil da Physical Appearance Perfectionism Scale 


\section{Validação da PAPS no Brasil}

em uma amostra de adultos de ambos os sexos, todos para praticantes de exercícios resistidos especificamente a musculação. Foi possível gerar evidencias limitadas de validade convergente, evidencias de validade discriminante e de confiabilidade interna. Algumas modificações foram necessárias para se obter um ajuste adequado da PAPS, embora essas modificações não tenham comprometido a estrutura teórica proposta originalmente. Esse foi o primeiro estudo transcultural da PAPS que temos conhecimento e por isso seus achados fazem contribuição para o aperfeiçoamento da avaliação do perfeccionismo da aparência.

Em relação à validade convergente, os valores das cargas fatoriais nos fatores preocupação com a imperfeição esperança de perfeição foram mais baixos que o desejável para alguns itens, o que comprometeu o valor da AVE final. Testamos um modelo mais reduzido em ajustes subsequentes, eliminando os itens 8 e 5.

\begin{tabular}{|c|c|c|c|c|c|c|c|c|}
\hline$\overline{\mathrm{MI}}$ & $\mathrm{gl}$ & $\chi^{2}$ & RMSEA & CFI & NFI & $\Delta \chi^{2}$ & $\Delta \mathrm{gl}$ & $\mathrm{p} \Delta \chi^{2}$ \\
\hline Configural & 62 & 142.15 & .069 & .94 & .91 & $\mathrm{~N} / \mathrm{A}$ & $\mathrm{N} / \mathrm{A}$ & $\mathrm{N} / \mathrm{A}$ \\
\hline Métrica & 73 & 183.75 & .075 & .96 & .93 & 41.6 & 6 & .001 \\
\hline Escalar & 91 & 218.41 & .073 & .91 & .87 & 34.66 & 18 & .01 \\
\hline Estrita & 104 & 239.08 & .066 & .91 & .86 & 20.67 & 13 & .08 \\
\hline
\end{tabular}

Nota: $M I=$ medida de invariância, $g l=$ graus de liberdade, $\chi^{2}=$ Qui-Quadrado Satorra-Bentler; RMSEA $=$ Root Mean Square Error of Approximation CFI $=$ Comparative Fit Index, NFI = Normed Fit Index, $\Delta \chi^{2}=$ delta Qui-Quadrado Satorra-Bentler; $\Delta g l=$ delta graus de liberdade; $p \Delta \chi^{2}$ nível de significância do valor de P para o teste de diferença Qui-Quadrado Satorra-Bentler.

Todavia, houve um ganho estatístico pequeno em relação à $\mathrm{AVE}$ e o instrumento ficou por demais empobrecido. Buscou-se entender então os motivos para tais resultados. De fato, as cargas fatoriais da versão brasileira são numericamente menores que o da versão original em Mandarim. A amostra brasileira é cerca de 4 vezes maior que a amostra chinesa empregada no estudo de desenvolvimento respectivamente 534 vs. 131 participantes no estudo 3, no qual se fez a CFA. Amostras maiores geram cargas fatoriais mais precisas e estáveis (Shevlin \& Miles, 1998; MacCallum, Widaman, Zhang, \& Hong, 1999), e nossa amostra pode ter revelado que são menores as contribuições dos itens para a explicação das variáveis latentes.

Outra explicação coerente versa sobre as diferenças culturais entre oriente e ocidente, já verificadas em alguns aspectos específicos - como autoestima, satisfação com o corpo, atratividade física, ansiedade social, estima corporal, vergonha, entre outros (Tasai, Ying \& Lee, 2001; Kowner, 2002; Swami \& Tovée, 2005; Jung \& Lee, 2006; Zhong et al., 2008) . O interessante é que há um processo de "aculturação" e globalização que aproxima os ideais de corpo e de beleza entre as sociedades ocidentais e orientais (Nielson, Reel, Galli, Crookston, \& Miyairi, 2013). O perfeccionismo em relação à aparência pode então ter algumas características específicas do oriente e ocidente, mas igualmente ter pontos comuns. Podese afirmar os pontos de convergência nesse estudo baseando-nos nos índices satisfatórios de ajuste obtidos, o que nos aponta para um ajuste geral da teoria aos dados (Hair et al., 2009). Ao mesmo tempo, a avaliação das cargas fatoriais e sua comparação com o estudo original (nas quais variaram de $.48 \mathrm{a} .84$ ) nos permite afirmar que essas variáveis observáveis contribuem menos entre Brasileiro que entre Chineses para a avaliação do perfeccionismo em relação à aparência. Este cenário pode parecer paradoxal - um ajuste satisfatório de modelo combinado com cargas fatoriais mais fracas. No entanto, é importante reconhecer que os índices de ajuste (por exemplo, GFI) refletem a adequação geral ou ajuste de todo o modelo de medição. Assim, um modelo pode realmente ter um bom ajuste, apesar de ter alguns aspectos específicos fracos.

Em relação à validade discriminante, a AVE de cada fator foi superior a variância compartilhada, o 


\section{Nogueira-Neves, A.; Francisco, J.N.; Corazza, J.F.; Carvalho, R.L.P..; Ferreira, L.}

que sugere que a semelhança dos itens em cada variável latente é superior à semelhança dos itens entre as variáveis latentes, logo, as variáveis latentes são distintas entre si (Fornell \& Larcker, 1981). Já a consistência interna da escala teve evidências satisfatórias nos dois parâmetros escolhidos, nos levando a concluir que a medida pode gerar dados confiáveis.

Cabe ainda salientar que no próprio estudo de desenvolvimento da PAPS (Yang \& Stoeber, 2012) não foi alcançado ajuste satisfatório para a amostra americana. Então, parece realmente que o modelo estrutural da PAPS ainda pode ser modificado e aprimorado. Essa evidencia do estudo original permite que discutamos três contribuições deste estudo para o posterior e contínuo desenvolvimento desse instrumento: a eliminação dos itens 1 e 2; a aceitação das covariâncias dos erros e a não invariância do modelo estrutural entre homens e mulheres.

Os itens 1 e 2 foram eliminados pois apresentaram carga fatorial muito baixas, em um modelo com ajuste insatisfatório. Para ajuste procedemos com a eliminação, baseando-nos nos possíveis motivos para baixas cargas fatoriais anteriormente citados. Uma segunda alteração feita no modelo original é a aceitação das covariâncias de erros entre os itens $9-8,3-9$ e $6-7$. A aceitação das covariâncias deve ser fundamentada teoricamente, e não apenas na expectativa de melhora de ajuste do modelo - razões estatísticas (Silvia \& MacCallum, 1988). Covariância de erros pode ocorrer especialmente por redundância de itens (de conteúdo ou por desejabilidade social) ou por omissão de um fator exógeno (quando dois indicadores têm uma mesma causa que não abordada no modelo). A desejabilidade social pode ser um fator descartado, uma vez que procurou-se diminuir as condições para sua ocorrência com a segurança de anonimidade, de participação voluntária e de um espaço privado para preenchimento das escalas. Não se mostra também plausível que um fator exógeno comum seja a causa das covariâncias aqui acatadas, pois a variável latente da PAPS está bem definida teoricamente. Analisadas uma a uma, a causa mais provável de sua ocorrência foi a semelhança de conteúdo dos itens. Essa constatação foi fundamentada pela análise do estudo de adaptação cultural da PAPS no Brasil (Ferreira et al., in press). Essa ação possibilitou assegurar que a semelhança de conteúdo existia originalmente e não havia sido criada pelo processo de tradução.
Quanto a não-invariância entre a amostra feminina e masculina, tal achado é uma contribuição realmente inédita, pois não foi averiguada nem no estudo original. Houve evidência somente da nãoinvariância configural - ou seja, para a amostra feminina e masculina a PAPS teve o mesmo modelo estrutural. Todavia, a não invariância métrica, escalar e estrita não puderam ser comprovadas. Isso aponta para a impossibilidade de comparar diretamente os escores feminino e masculino. Aponta igualmente para uma possível igualde teórica do perfeccionismo relacionado à aparência entre homens e mulheres, mas com diferentes atitudes, crenças e comportamentos observáveis. Recomendamos que essa análise seja feita em outras amostras, o que pode gerar mais evidências para uso da PAPS. Ao mesmo tempo, estudos qualitativos podem evidenciar diferenças e semelhanças do perfeccionismo da aparência entre homens e mulheres, contribuindo para posterior aperfeiçoamento da PAPS.

Por fim, devemos comentar as evidências da validade nomológica, obtida através da análise de correlação entre os escores dos fatores da PAPS da EDS e do escore da SPAS. Houve confirmação parcial da associação esperada entre o perfeccionismo da aparência e a dependência do exercício (Hagan \& Hausenblas, 2003; Mohammed, 2017). A exceção foram os fatores Abstinência e Continuidade, que em última instancia, tratam de atitudes mais diversas - os sintomas de não fazer exercício e a necessidade de cada vez mais exercício. A associação esperada entre ansiedade físico social e perfeccionismo (Sabiston , Pila, Pinsonnault-Bilodeau, \& Cox, 2014) foi comprovada tanto para homens quanto para mulheres.

Apesar das contribuições que este estudo faz para a mensuração do perfeccionismo da aparência, ele tem limitações. Primeiro, deve-se considerar que a amostra foi composta exclusivamente por praticantes de exercício resistido (musculação) e assim, as diferenças e modificações aqui feitas podem pertencer a essa amostra de referencia unicamente. Por isso, é necessário avaliar a PAPS em outros grupos e outras amostras brasileiras - e mesmo internacionais - para que a teoria e a medida do perfeccionismo da aparência possam continuar evoluindo. Segundo, apesar de termos avaliados dois aspectos teoricamente previsto como associados ao perfeccionismo da aparência - dependência do exercício e ansiedade físico social - outros não o foram. Transtornos alimentares, dismorfia muscular, dismorfia corporal, 


\section{Validação da PAPS no Brasil}

insatisfação com o corpo, autoestima, comportamentos de checagem e evitação corporal são outros aspectos que devem ser futuramente avaliados e discutidos juntamente com o perfeccionismo em relação à aparência. Pode-se gerar modelos estruturais que vão nos levar ao melhor entendimento desse constructo. Terceiro, é preciso que a medida evolua para grupos nos quais as modificações da aparência são visíveis, como por exemplo, pessoas com queimaduras, com lipodistrofia, vitiligo e mancha na pele em geral. Isso nos permitirá discutir e compreender aspectos dessa experiência de modificação da aparência no perfeccionismo, do estigma social, da vergonha e culpa, permitindo avançar nas proposições teóricas e na avaliação.

\section{CONCLUSÕES}

O presente estudo disponibiliza ao pesquisador brasileiro a Physical Appearance Perfectionism Scale. Para o pesquisador internacional, insights e ideias sobre as diferenças culturais e a avaliação desse constructo.

\section{APLICAÇÕES PRÁTICAS}

Como o perfeccnionismo mal adaptado e o perfeccionismo da aparência já foram sistematicamente associados aos transtornos alimentares, dismorfia muscular e excesso de exercícios, parece ser relevante ter dados das atitudes do praticante de exercício físico em relação a esse constructo. A PAPS é útil, em especial, para acompanhamento de intervenções com exercícios físico voltados para promoção da saúde, na abordagem do psicólogo da saúde e/ou do exercício, para avaliação do impacto das experiências vividas no programa de exercícios no perfeccionismo da aparência.

\section{REFERÊNCIAS}

1. Adams, G., Truner, H., \& Bucks, R. (2005). The experience of body dissatisfaction in men. BodyImage, 2, 271-283. doi:10.1016/j.bodyim.2005.05.004

2. Alchieri, J. C., Gouveia, V. V., de Oliveira, I. C. V., \& Diógenes, E. (2015). Exercise Dependence
Scale: adaptação e evidências de validade e precisão. Jornal Brasileiro de Psiquiatria, 64(4), 279-87.doi: 10.1590/0047-2085000000090

3. Bieling, P. J., Israeli, A. L., \& Antony, M. M. (2004). Is perfectionism good, bad, or both? Examining models of the perfectionism construct. Personality and Individual Differences, 36, 13731385. doi:10.1016/S0191-8869(03)00235-6.

4. Brown, T. A. (2014). Confirmatory factor analysis for applied research. Guilford Publications.

5. Cash, T. F., \& Duncan, N. C. (1984). Physical attractiveness stereotyping among BlackAmerican college students. Journal of Social Psychology, 122, 71-77.doi: 10.1080/00224545.1984.9713459

6. Cash, T. F., \& Smolak, L. (Eds.). (2011). Body image: A handbook of science, practice, and prevention. Guilford Press.

7. Cockell, S. J., Hewitt, P. L., Seal, B., Sherry, S. B., Goldner, E. M., et al. (2002). Trait and selfpresentational dimensions of perfectionism among women with anorexia nervosa. Cognitive Therapy and Research, 26, 745-758. doi: 10.1023/A:1021237416366

8. Davis, C., \& Scott-Robertson, L. (2000). A psychological comparison of female with anorexia nervosa and competitive male bodybuilders: Body shape ideals in the extreme. Eating Behaviors, 1, 33-46. doi: 10.1016/S1471-0153(00)00007-6

9. Deaux, K., \& Lewis, L. L. (1984). Structure of gender stereotypes: Interrelationships among components and gender label. Journal of Personality and Social Psychology, 46, 991-1004. doi: 10.1037/0022-3514.46.5.991

10. Dermer, M., \& Thiel, D. I. (1975). When beauty may fail. Journal of Personality and Social Psychology, 31, 1168-1176. doi: 10.1037/h0077085

11. Dion, K., Berscheid, E., Walster, E. (1972). What is beautiful is good. Journal of Personality and Social Psychology, 24, 285-290. doi: 10.1037/h0033731

12. Downs, D. S., Hausenblas, H. A., \&Nigg, C. R. (2004). Factorial validity and psychometric examination of the Exercise Dependence ScaleRevised. Measurement in physical education and 


\section{Nogueira-Neves, A.; Francisco, J.N.; Corazza, J.F.; Carvalho, R.L.P..; Ferreira, L.}

exercise science, $8(4), \quad$ 183-201.doi: $10.1207 / \mathrm{s} 15327841 \mathrm{mpee} 0804 \_1$

13. Ferreira, L., Corazza, J. F., Francisco, J. N., \& Neves, A. N. (in press). Tradução e adaptação cultural da Escala de Perfeccionismo para Aparência Física (PAPS) para a língua portuguesa no Brasil. Revista Brasileira de Ciências do Esporte.doi: 10.1016/j.rbce.2018.03.002

14. Fornell, C. \&Larcker, D. (1981). Structural equation models with unobservable variables and measurement error. Journal of Marketing Research, 18, 39-50.doi: 10.2307/3150980

15. Garson, G. D. (2006). PA 765 Statnotes: an online Textbook. Retrieved March 13, 2011, from http://www2.chass.ncsu.edu/garson/pa765/statnot e.html.

16. Gillen, H. B. (1981). Physical attractiveness: A determinant of two types of goodness. Personality and Social Psychology Bulletin, 7, 277-281. doi: 10.1177/014616728172015

17. Grogan, S. (2008). Body image: Understanding body dissatisfaction in men, women and children. London: Routledge.

18. Gulker, M.G., Laskis, T.A., \&Kuba, S.A. (2001). Do excessive exercisers have a higher rate of obsessive-compulsive symptomology? Psychology, Health, and Medicine, 6, 387-398. doi: 10.1080/13548500126535

19. Haase, A. M., Prapavessis, H., \& Owens, R. G. (2002). Perfectionism, social physique anxiety and disordered eating: a comparison of male and female elite athletes. Psychology of Sport and Exercise, 3, 209-222. doi:10.1016/S14690292(01)00018-8.

20. Hagan, A. L. \& Hausenblas, H. A. (2003). The relationship between exercise dependence symptoms and perfectionism. American Journal of Health Studies, 18, 133-137.

21. Hair, Jr., J. F., Anderson, R. E., Tatham, R. L. y Black, W. B. (2009) Multivariate data analysis. Upper Saddle River, NJ: Prentice Hall.

22. Hanstock, T. L., \& O’Mahony, J. F. (2002). Perfectionism, acne and appearance concerns. Personality and Individual Differences, 32, 13171325. doi:10.1016/s0191-8869(01)00120-9.
23. Hardman, A. E., \& Stensel, D. J. (2009). Physical activity and health: The evidence explained. Routledge.

24. Hart, E., Leary, M.R. \& Rejesky, W.J. (1989).The measurement of social physique anxiety.Journal of Sport and Exercise Psychology, 11, 94-104.

25. Hennessy, M. H., Fishbein, M., Curtis, B., \& Barrett, D. (2008). Confirming preferences or collecting data? Information search strategies and romantic partner selection. Psychology, health\& medicine, 13(2), 202-221.doi: $10.1080 / 13548500701246010$

26. Hewitt, P. L., Sherry, S. B., Flett, G. L., \& Shick, R. (2003). Perfectionism and cosmetic surgery. Plastic \& Reconstructive Surgery, 112, 346. doi: 10.1097/01.PRS.0000067100.50738.73

27. Jöreskog, K. \& Sörbom, D. (1999). Prelis $^{T M} 2$ : User's reference guide. Chicago, IL: Scientific Software International.

28. Jung, J., \& Lee, S. H. (2006). Cross- cultural comparisons of appearance self- schema, body image, self- esteem, and dieting behavior between Korean and US women. Family and Consumer Sciences Research Journal, 34(4), 350-365.doi: 10.1177/1077727X06286419

29. Kaplan, D. (2000). Structural Equations modeling: foundations and extensions. Thousand Oaks: Sage Publications.

30. Kowner, R. (2002). Japanese body image: Structure and esteem scores in a cross-cultural perspective. International Journal of Psychology, 37(3), 149-159.doi: 10.1080/00207590143000298

31. MacCallum, R. C., Widaman, K. F., Zhang, S., \& Hong, S. (1999). Sample size in factor analysis. Psychological methods, 4(1), 84.doi:10.1037/1082-989X.4.1.84

32. Marôco, J. (2014). Análise de equações estruturais (2 ${ }^{\mathrm{a}}$ ed.). Lisboa: ReportNumber

33. Mohammed, S. (2017). The role of perfectionism and shame in understanding excessive exercise tendencies [Doctoral dissertation]. Laurentian University of Sudbury, Ontário, Canada

34. Nascimento Junior, J. R. A. D., Vissoci, J. R. N., Lavallee, D., \& Vieira, L. F. (2015). Adaptation 


\section{Validação da PAPS no Brasil}

and validation of the Sport Multidimensional Perfectionism Scale-2 (SMPS-2) for the Brazilian sport context. Motriz: Revista de Educação Física, 21(2), 125-136.doi: 10.1590/S198065742015000200003

35. Neves, A.N., Neves, A.B., Zanetti, M. C, Brandão, M. R.F., \& Ferreira, L. (2018). Validação psicométrica da social physique anxiety scale no Brasil. Revista Iberoamericana de Psicología Del Ejercicio y el Deporte, 13(2).

36. Nielson, H. E., Reel, J. J., Galli, N. A., Crookston, B. T., \& Miyairi, M. (2013). Body image and westernization trends among Japanese adolescents. Health Educator, 45(1), 4.

37. Nunnally J C. Psychometric theory. New York: McGraw Hill, 1978.

38. Ruggiero, G. M., Levi, D., Ciuna, A., \& Sassaroli, S. (2003). Stress situation reveals an association between perfectionism and drive for thinness. International Journal of Eating Disorders, 34(2), 220-226. doi:10.1002/eat.10191

39. Sabiston, C. M., Pila, E., Pinsonnault-Bilodeau, G., \& Cox, A. E. (2014). Social physique anxiety experiences in physical activity: a comprehensive synthesis of research studies focused on measurement, theory, and predictors and outcomes. International Review of Sport and Exercise Psychology, 7(1), 158-183.doi: 10.1080/1750984X.2014.904392

40. Schwartz, J. P., \& Tylka, T. L. (2008). Exploring entitlement as a moderator and mediator of the relationship between masculine gender role conflict and men's body esteem. Psychology of Men \& Masculinity, 9, 67-81. doi: 10.1037/15249220.9.2.67

41. Shevlin, M., \& Miles, J. N. (1998). Effects of sample size, model specification and factor loadings on the GFI in confirmatory factor analysis. Personality and Individual differences, 25(1), 85-90. doi: $10.1016 / \mathrm{S} 0191-$ 8869(98)00055-5

42. Silvia, E. S. M. \& Maccallum, R. C. (1988). Some factors affecting the success of specification searches in covariance structure modeling. Mutlivariate Behavioral Research, 23, 297326.doi: 10.1207/s15327906mbr2303_2
43. Stoeber, J., \& Yang, H. (2015). Physical appearance perfectionism explains variance in eating disorder symptoms above general perfectionism. Personality and Individual Differences, 86, 303-307. doi: 10.1016/j.paid.2015.06.032

44. Swami, V., \& Tovée, M. J. (2005). Female physical attractiveness in Britain and Malaysia: A cross-cultural study. Body image, 2(2), 115128.doi: 10.1016/j.bodyim.2005.02.002

45. Tsai, J. L., Ying, Y. W., \& Lee, P. A. (2001). Cultural predictors of self-esteem: A study of Chinese American female and male young adults. Cultural Diversity and Ethnic Minority Psychology, 7(3), 284.doi: 10.1037//10999809.7.3.284

46. Vigarello, G. (2005). A história da Beleza. Rio de Janeiro: Ediouro

47. Yang, H., \& Stoeber, J. (2012). The physical appearance perfectionism scale: Development and preliminary validation. Journal of Psychopathology and Behavioral Assessment, 34(1), 69-83.doi: 10.1007/s10862011-9260-7

48. Zhong, J., Wang, A., Qian, M., Zhang, L., Gao, J., Yang, J., ... \& Chen, P. (2008). Shame, personality, and social anxiety symptoms in Chinese and American nonclinical samples: a cross- cultural study. Depression and Anxiety, 25(5), 449-460.doi: 10.1002/da.20358 